\title{
Visualização de Escoamentos pelos Métodos Ópticos Shadowgraph e Schlieren
}

\author{
Flow Visualization using the Shadowgraph and Schlieren Optical Methods
}

\author{
Danilo Almeida Machado*10 ${ }^{*}$ Fernando de Souza Costa ${ }^{1}$, Dermeval Carinhana Junior ${ }^{2}$, Antonio \\ Carlos de Oliveira ${ }^{2}$
}

${ }^{1}$ Instituto Nacional de Pesquisas Espaciais, Laboratório Associado de Combustão e Propulsão, Cachoeira Paulista, Brasil.
${ }^{2}$ Instituto de Estudos Avançados, Divisão de Aerotermodinâmica e Hipersônica, São José dos Campos, Brasil.

Recebido em 20 de abril de 2020. Revisado em 18 de maio de 2020. Aceito em 23 de maio de 2020.

\begin{abstract}
A utilização de experimentos é uma das formas mais eficientes para o ensino de física, uma vez que os alunos tornam-se mais motivados quando conceitos teóricos fundamentais são associados a aplicações tecnológicas. O desenvolvimento de carros, aeronaves e foguetes, por exemplo, requer o conhecimento detalhado dos escoamentos de fluidos ao redor e no interior desses veículos. O presente artigo descreve os fundamentos da óptica geométrica em meios heterogêneos e apresenta as técnicas ópticas shadowgraph e schlieren que são amplamente utilizadas em centros de pesquisa, universidades e empresas no estudo da aerodinâmica e dos escoamentos em geral. Esses métodos ópticos são baseados na deflexão dos raios de luz causada por variações na massa específica do escoamento. O método schlieren-Toepler é apresentado e discutido em detalhes, derivando-se equações para o contraste, sensibilidade e o alinhamento óptico. Uma implementação do método shadowgraph direto para uso em sala de aula é demonstrada e, ao longo do artigo, são apresentadas diversas imagens obtidas pelos métodos ópticos descritos. Palavras-chave: diagnóstico não-intrusivo, imageamento de escoamento, schlieren, shadowgraph, meios heterogêneos.
\end{abstract}

The use of experiments is one of the most efficient ways to teach physics, since students become more motivated when fundamental theoretical concepts are associated with technological applications. The development of cars, aircraft and rockets, for example, requires detailed knowledge of the flow of fluids around and inside these vehicles. This article describes the fundamentals of geometric optics in heterogeneous media and presents the shadowgraph and schlieren optical techniques that are widely used in research centers, universities and companies in the study of aerodynamics and flows in general. These optical methods are based on the deflection of the light rays caused by variations in the flow density. The schlieren-Toepler method is discussed in detail, deriving equations for contrast, sensitivity and optical alignment. An implementation of the direct shadowgraph method for classroom use is demonstrated and, throughout the article, several images obtained by the described optical methods are presented. Keywords: non-intrusive diagnostic, flow imaging, schlieren, shadowgraph, heterogeneous media.

\section{Introdução}

Observações e experimentos são indispensáveis no desenvolvimento da Física. A teoria é importante, mas somente se as suas consequências puderem ser verificadas por observações ou por meio de experimentos. O ensino da Física se torna também mais eficaz com o auxílio de atividades experimentais em sala de aula [1].

Independente da metodologia da atividade experimental, a correlação entre o ensino de conceitos básicos e experimentais com aplicações tecnológicas é uma das formas mais atraentes de motivar alunos para aprender física $[2]$.

Com relação ao ensino de óptica geométrica, normalmente os livros-texto de Física para o ensino médio e a graduação iniciam com uma abordagem sobre a na-

*Endereço de correspondência: danilo.machado@inpe.br tureza da luz, seguida de uma descrição dos fenômenos de propagação, reflexão e refração da luz e depois pela apresentação de conceitos sobre lentes e espelhos, bem como da formação de imagens, penumbras e sombras.

O presente trabalho apresenta os aspectos principais da óptica geométrica com vistas a descrever os métodos ópticos shadowgraph e schlieren os quais são baseados na deflexão da luz por gradientes de massa específica ou densidade do meio. Tais métodos permitem a observação dos escoamentos de gases, normalmente invisíveis a olho nu. O termo schlieren tem sua origem no idioma alemão e significa "heterogeneidades", resultantes de variações de densidade em um meio, enquanto shadowgraph tem origem no idioma inglês e significa imagem pela sombra.

Existem diversas variantes dos métodos schlieren e shadowgraph, sendo algumas delas descritas nesse trabalho. Os métodos schlieren permitem a determinação da geo- 
metria e das distribuições das velocidades, temperaturas e de massas específicas de um escoamento, enquanto os métodos shadowgraph possibilitam observar a geometria e a distribuição de velocidades de um escoamento.

\section{Métodos de Visualização de Escoamentos}

O uso dos métodos ópticos shadowgraph e schlieren para o diagnóstico não intrusivo de escoamentos remonta ao início do século XIX. Ao longo do tempo, esses métodos foram se tornando cada vez mais sensíveis e com melhor resolução 3 .

A visualização de escoamentos por métodos shadowgraph ou schlieren tem sido uma das formas mais simples e utilizadas para estudar e analisar de forma qualitativa a estrutura de um escoamento real, bem como comparar com a estrutura gerada por métodos computacionais [3], [4]. Nos últimos 15 anos esses métodos, basicamente qualitativos, sofreram uma profunda transformação, tornando-se também métodos quantitativos graças ao uso de câmeras de alta resolução e de alta velocidade, bem como graças ao processamento de imagens por softwares [5]. Com o uso de computadores para processamento de imagens é possível determinar as distribuições de velocidade, temperatura e de massa específica dos escoamentos usando métodos ópticos. Por consequência, os métodos shadowgraph e schlieren voltaram ao estado da arte na área de diagnósticos ópticos de escoamentos.

Como mencionado anteriormente, existem variações dos métodos shadowgraph e schlieren. Por exemplo, a técnica Schlieren Colorido (Rainbow Schlieren) permite a determinação dos campos bidimensionais de densidade e de temperatura de escoamentos 6. 7], enquanto a técnica de Velocimetria por Imagem Schlieren (Schlieren Image Velocimetry - SIV) possibilita medir o campo bidimensional de velocidade de escoamentos 8, 9]. Em comum às técnicas SIV e Schlieren Colorido está a possibilidade de se adotar um arranjo óptico do tipo Toepler para aquisição das imagens que tem como principais vantagens a sensibilidade, a facilidade de alinhamento, baixo custo dos seus componentes ópticos e possibilidade de imageamento de escoamentos de diferentes tamanhos [10].

\section{O Experimento de Hooke}

Hooke foi o primeiro cientista a realizar uma discussão aprofundada sobre a refração da luz causada por variações da densidade do ar 3 . O fascínio de Hooke com a refração atmosférica levou-o a estabelecer a óptica dos meios heterogêneos como um novo campo de investigação científica. Para demonstrar seus conceitos, propôs um experimento utilizando uma lente e duas chamas cujas posições relativas são mostradas na Figura 1. De acordo com suas observações, parte da luz proveniente da primeira vela é refratada pela chama de uma segunda vela. Ao passar por uma lente, os raios refratados formam uma imagem no olho do observador revelando a formação de uma pluma convectiva acima da chama. Com esse experimento, Hooke foi o primeiro a observar o fenômeno associado à perturbação dos gases pelo calor, de uma forma metódica, descrita a partir de princípios ópticos estabelecidos segundo a óptica geométrica. O arranjo de Hooke é um método shadowgraph primitivo.

A Figura 2 mostra a imagem shadowgraph das chamas e plumas convectivas de duas velas, empregando-se um projetor multimídia como fonte de luz.

Observam-se contornos brilhantes indicando as regiões de maior incidência de luz, resultantes de uma maior curvatura do campo de densidades do escoamento. A chama de uma vela é do tipo difusiva, ocorrendo reação somente em uma região de espessura bem fina na periferia, sem reação no interior da chama. Nessa zona fina de reação a temperatura alcança valores elevados e ocorre uma diminuição na massa específica ou densidade, uma vez que a pressão se mantém aproximadamente constante e igual a 1 atm. Portanto, a densidade é bem menor na zona de reação da chama do que no ar ao redor, formandose um gradiente de densidades cujo máximo ocorre na periferia da chama.

No experimento de Hooke foram lançadas as sementes para o desenvolvimento das técnicas de visualização de

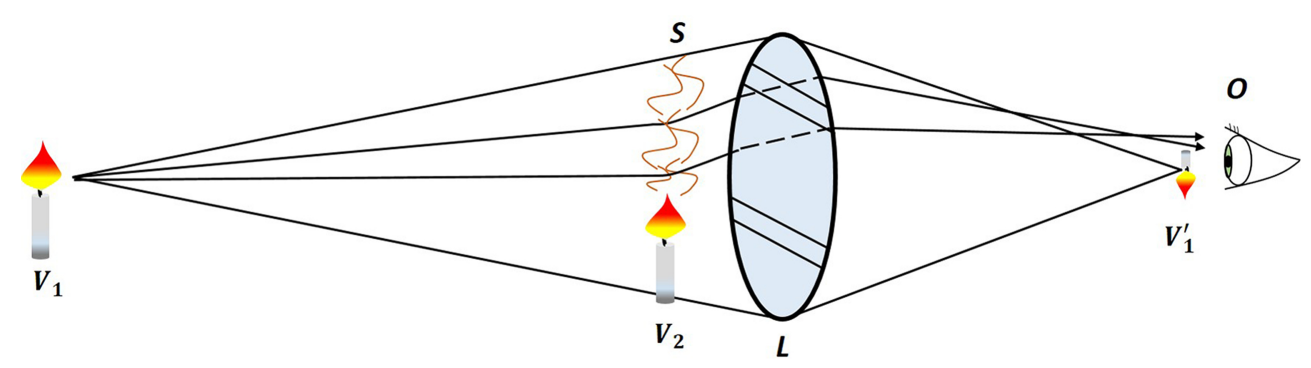

Figura 1: Arranjo óptico adotado por Hooke para observar a chama de uma vela. $V_{1}$ é a vela de fonte de luz, $V_{2}$ é a vela na região de testes, $S$ é a região de testes, Lé a lente, $V_{1}^{\prime}$ é a imagem invertida formada de $V_{1}$ e $O$ é onde se localiza o olho do observador. Na prática as distâncias da vela $V_{1}$ e do olho $O$ para a lente $L$ são relativamente maiores que no esquema mostrado, de forma que as imagens da vela $V_{1}$ e $V_{2}$ ficam próximas. 


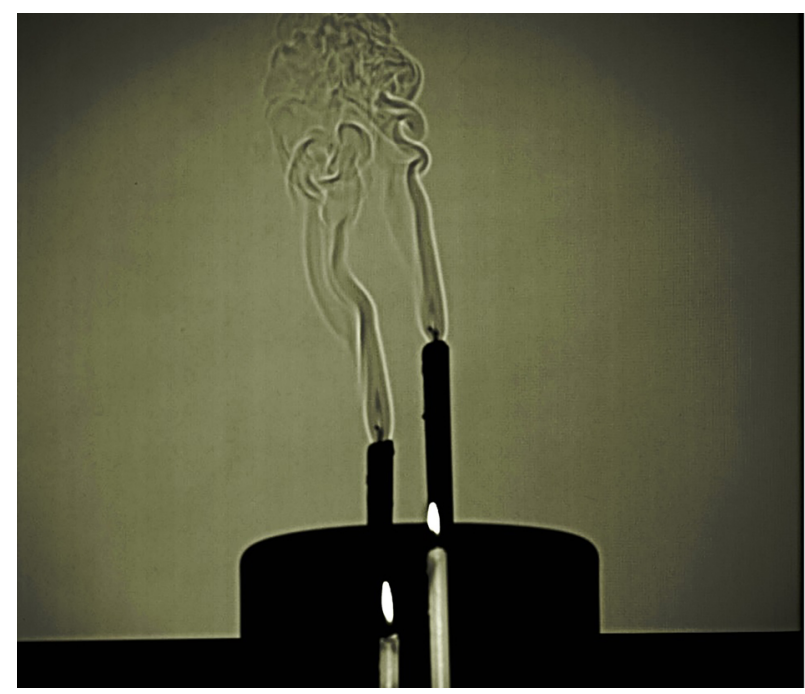

Figura 2: Imagem shadowgraph obtida em sala de aula das chamas e plumas convectivas de duas velas. Fonte: os autores.

escoamentos por meios ópticos, no caso através da técnica shadowgraph.

Em 1864, August Toepler inventou um novo método, denominado schlieren, para visualização e registro das imagens resultantes de gradientes da densidade do escoamento sob estudo 3. Toepler desenvolveu um procedimento detalhado de como projetar um arranjo de alta sensibilidade para visualização de escoamentos e foi o primeiro cientista a observar ondas de choque que surgem nos escoamentos supersônicos 11 .

O método desenvolvido por Toepler é, até os dias de hoje, um dos métodos mais utilizados na visualização de escoamentos, apresentando grande sensibilidade de visualização da imagem e facilidade de montagem do arranjo óptico [3], [12]]. Os olhos humanos e as câmeras de vídeo ou fotográficas comuns não conseguem discernir as diferenças de fase em um feixe de luz. Podem-se ver apenas a amplitude e o contraste de cores. Se fosse possível ver a fase da luz, novas perspectivas se abririam para nossa visão. Tal é a importância do método óptico schlieren: ele transforma as diferenças de fase em amplitude 3$]$. Em outras palavras, com o uso do método schlieren é possível observar uma vasta gama de fenômenos invisíveis a olho nu.

Na seção a seguir serão feitas considerações sobre a refração da luz em meios heterogêneos e serão descritos os desvios angulares da luz e as características dos sistemas shadowgraph e schlieren, usando a formulação teórica, baseada na óptica geométrica, descrita por Settles [3] e por Liepmann and Roshko [13.

\section{Propagação da Luz em Meios Heterogêneos}

A sensibilidade dos métodos schlieren e shadowgraph depende da variação do índice de refração entre os meios e das características dos instrumentos ópticos utilizados no arranjo. A parte da física que descreve a variação do índice de refração em meios gasosos é a óptica dos meios heterogêneos. A luz se propaga uniformemente através de meios homogêneos seguindo um caminho retilíneo. Por outro lado, ao passar por meios heterogêneos a luz sofre desvios decorrentes da não uniformidade do índice de refração. Quando se observa o céu, tem-se a impressão de que as estrelas piscam. Este efeito, denominado de cintilação astronômica, é oriundo da não homogeneidade da atmosfera terrestre. Se a atmosfera terrestre fosse uniforme, as estrelas não cintilariam e seu brilho seria estático, como o observado no espaço por telescópios e astronautas. Porém, como a atmosfera varia com a altitude, possuindo diferentes valores de temperatura, pressão e densidade, esses gradientes causam uma variação do índice de refração do meio. De forma simplificada pode-se entender esta cintilação observando que as estrelas são fontes de luz puntiformes extremamente distantes. Isto faz com que os seus raios sejam paralelos na atmosfera. O feixe de luz de uma estrela atravessa a camada da atmosfera que possui, aproximadamente, $100 \mathrm{~km}$ de espessura. Esse feixe estreito sofre desvios nesse trajeto, produzindo as variações de posição nos olhos e dando a sensação da cintilação. Esse mesmo efeito não ocorre com os planetas porque estes são fontes em forma de disco e seus feixes não são paralelos. Assim, o efeito de desvio de uma borda do disco é compensado pelo outro lado e a intensidade média permanece constante.

A luz diminui ou aumenta a sua velocidade ao passar de um meio a outro e, por consequência, o seu comprimento de onda varia na mesma proporção, uma vez que a frequência da luz não é afetada pela mudança do meio [3]. O índice de refração da luz em um meio $(n)$ relaciona a velocidade da luz no vácuo à velocidade da luz nesse meio, sendo dado por:

$$
n=\frac{c_{0}}{c}=\frac{\lambda_{0}}{\lambda}
$$

onde $c$ é a velocidade da luz no meio, $c_{0}$ é a velocidade da luz no vácuo que vale aproximadamente $2,998 \times 10^{8} \mathrm{~m} / \mathrm{s}$, $\lambda$ é o comprimento de onda da luz no meio e $\lambda_{0}$ é o comprimento de onda da luz no vácuo. Em um meio gasoso, o índice de refração é proporcional à massa específica do gás, de modo que:

$$
n-1=k \rho
$$

em que $\rho$ é a massa específica do gás e $k$ é o coeficiente de Gladstone-Dale, equivalente a, aproximadamente, 0,23 $\mathrm{cm}^{3} / \mathrm{g}$, para o ar a $0{ }^{\circ} \mathrm{C}$ e a $1 \mathrm{~atm}$, no espectro visível. Para os demais gases, o coeficiente de Gladstone-Dale pode variar de 0,1 a 1,5 3]. A densidade ou massa específica de um gás pode ser alterada por variações de pressão ou temperatura, o que implica em uma variação no índice de refração do gás $(n)$. Gladstone e Dale foram os primeiros a propor uma relação entre a densidade e o índice de refração de um gás, essa relação ficou conhecida como coeficiente de Gladstone-Dale. 
O índice de refração dos gases comuns varia apenas na terceira ou quarta casa decimal. Por exemplo, o oxigênio, a 1 atm e $25{ }^{\circ} \mathrm{C}$, possui um índice de refração $n=1,000271$ para o comprimento de onda de $589,30 \mathrm{~nm}$, conhecido como a linha $D$ da emissão do sódio, correspondente à cor amarela. Já o nitrogênio, nas mesmas condições, tem $n=1,000273$ [14]. Para o hexafluoreto de enxofre tem-se $n=1,000783$, sendo esse um dos maiores valores para os gases, o que torna fácil sua observação pelo sistema schlieren [15]. O parâmetro $k$ diminui ligeiramente com o aumento do comprimento de onda da luz. Portanto, o índice de refração é maior para valores de $\lambda$ menores, de modo que as variações no índice de refração são mais intensas para raios ultravioleta do que no espectro visível.

A refratividade de um gás $(n-1)$ depende da composição do gás, temperatura, densidade e do comprimento de onda da radiação incidente $(\lambda)$. Essas grandezas podem ser relacionadas pela equação de estado do gás perfeito:

$$
n-1=\frac{k P M}{R_{0} T}
$$

em que $T$ é a temperatura, $M$ é a massa molar, $P$ é a pressão absoluta e $R_{0}$ é a constante universal dos gases. Variações de temperatura, densidade e pressão em um gás geram heterogeneidades no índice de refração de uma massa fluida. Por essa razão, um raio de luz ao atravessar a pluma de uma vela tem a sua trajetória modificada.

Considerando um raio de luz monocromático que atravessa a superfície de separação de dois meios transparentes com índices de refração $n_{1}$ e $n_{2}$, o ângulo do raio incidente $\theta_{1}$ e o ângulo do raio transmitido $\theta_{2}$ (ângulo de refração), medidos com respeito à normal [16], são relacionados pela Lei de Snell, dada por:

$$
n_{1} \operatorname{sen} \theta_{1}=n_{2} \operatorname{sen} \theta_{2}
$$

Conforme a Lei de Snell, quando um raio de luz passa de um meio para outro meio com índice de refração maior, $n_{2}>n_{1}$, então $\theta_{2}<\theta_{1}$, ou seja, o ângulo de refração $\theta_{2}$ será menor que o ângulo de incidência $\theta_{1}$. E se um raio de luz passa de um meio a outro meio com índice de refração menor, $n_{2}<n_{1}$, então $\theta_{2}>\theta_{1}$, ou seja, o ângulo de refração $\theta_{2}$ será maior que o ângulo de incidência $\theta_{1}$.

A Figura 3 mostra o comportamento dos raios de luz ao atravessar a interface de meios com diferentes índices de refração, no caso ar e água. Na Figura $3 a$, uma lanterna está suspensa no ar e emite um feixe de luz na direção da água, formando um ângulo $\theta_{1}$ com a normal (vertical). Ao atingir a superfície da água o feixe de luz forma um ângulo $\theta_{2}<\theta_{1}$ com a normal. Na Figura $3 b$, a lanterna está dentro d'água e emite um feixe de luz para cima na direção do ar, formando um ângulo $\theta_{1}$ com a vertical. Ao atingir a superfície da água o feixe de luz forma um ângulo $\theta_{2}>\theta_{1}$ com a normal.

Quando um meio apresenta uma variação contínua do índice de refração, a trajetória de um raio de luz que se propaga nesse meio apresenta uma curvatura que depende diretamente do gradiente do índice de refração. Em termos matemáticos, a curvatura é a variação do vetor tangente de uma curva em relação ao seu comprimento e o raio de curvatura é definido como o inverso da curvatura. A equação da curvatura de um raio de luz será derivada a seguir, de forma simplificada, através da óptica geométrica, considerando-se primeiramente um meio com variação do índice de refração em apenas uma direção, com base na abordagem apresentada por Liepmann e Roshko [13.

A Figura 4 mostra um meio cuja densidade ou massa específica é crescente ao longo da direção y e, portanto, apresenta variação crescente do índice de refração na direção $y$. Na Figura $4 a$ é mostrado um conjunto de raios de luz, denotados pela letra $r$, e respectivas posições das frentes de onda ao longo do tempo, denotadas pela letra $s$, que indicam a propagação da luz. Na Figura $4 b$ é mostrado um elemento da malha formada pelos raios de luz $r_{1}$ e $r_{2}$ e pela frente de onda que se desloca entre as posições $s_{1}$ e $s_{2}$ durante um intervalo de tempo $\Delta t$ dado

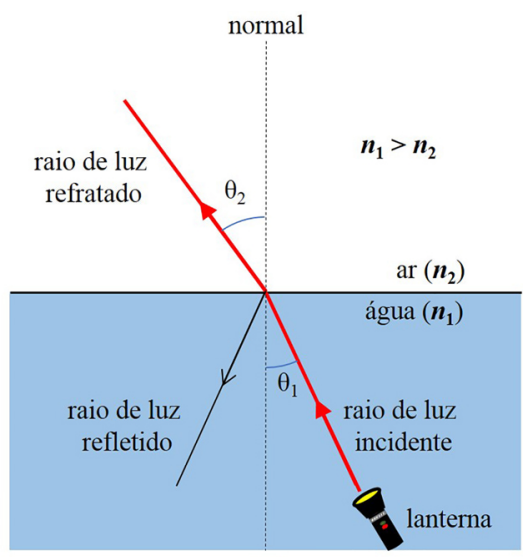

(b)

Figura 3: Refração da luz ao atravessar a interface de dois meios. 


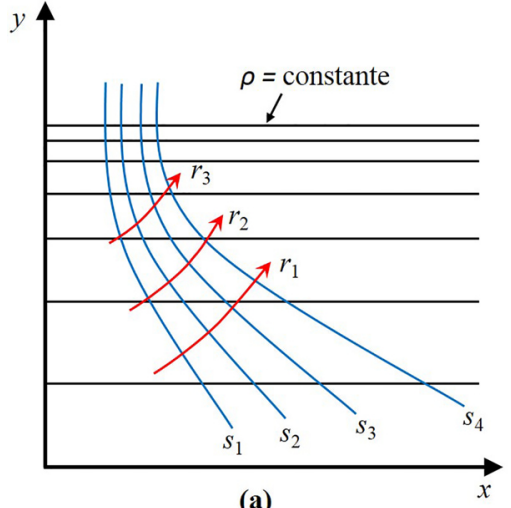

(a)

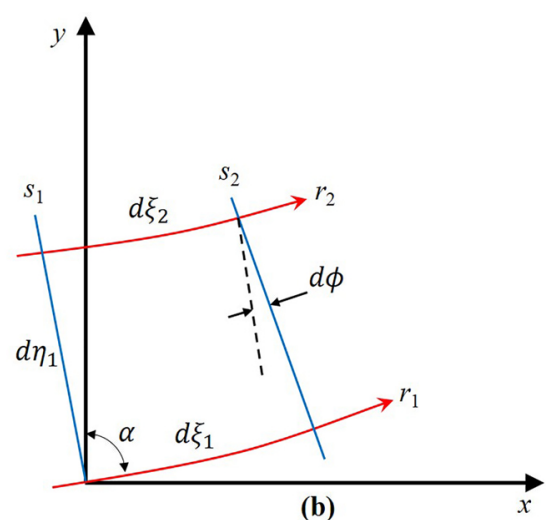

(b)

Figura 4: Refração de raios de luz em um campo de densidade unidimensional. Fonte: adaptada de Liepmann e Roshko [14].

por:

$$
\Delta t=\frac{d \xi_{1}}{c_{1}}
$$

onde $c_{1}$ é a velocidade do raio $r_{1}$ e $d \xi_{1}$ é a distância percorrida pela frente de onda do raio $r_{1}$.

Como a densidade do meio por onde passa o raio $r_{2}$ é maior que a densidade do meio por onde passa o raio $r_{1}$, então a velocidade do raio de luz $r_{1}$ é maior que a velocidade do raio de luz $r_{2}$. Isto resulta em um giro da frente de onda igual a

$$
d \phi=\frac{\left|c_{2} \Delta t-c_{1} \Delta t\right|}{d \eta_{1}}
$$

onde $d \eta_{1}$ é a distância inicial entre os raios de luz $r_{1}$ e $r_{2}$. O raio de luz $r_{1}$ é girado do mesmo ângulo $d \phi$ e o seu raio de curvatura $R$ é dado por:

$$
\frac{1}{R}=\frac{d \phi}{d \xi_{1}}=\frac{d}{d \xi_{1}}\left[\frac{\left|c_{2}-c_{1}\right| \Delta t}{d \eta_{1}}\right]
$$

Substituindo $d \xi_{1}=c_{1} \Delta t$ e tomando $d c=\left|c_{2}-c_{1}\right|$ fica

$$
\frac{d \phi}{d \xi}=\frac{1}{c}\left|\frac{d c}{d \eta}\right|=\frac{1}{n}\left|\frac{d n}{d \eta}\right|
$$

onde o subscrito 1 foi omitido para se considerar um raio de luz qualquer.

No caso de um raio de luz se propagando em um campo de densidade tridimensional, pode-se escrever:

$$
\frac{1}{R}=\operatorname{sen}(\alpha) \frac{|\nabla n|}{n}
$$

onde $\nabla n$ é o vetor gradiente de refração do meio e $\alpha$ é o ângulo entre este vetor e o raio de luz. Verifica-se, portanto, que a curvatura $(=1 / R)$ de um raio luz ocorre na direção da densidade crescente.

Quando as variações do índice de refração ocorrem predominantemente nas direções $x$ e $y$, ocorre a deflexão dos raios de luz nessas direções, conforme mostra a Figura 5 .

As componentes da deflexão são calculadas por:

$$
\varepsilon_{x}=\int d \phi_{x} \quad ; \quad \varepsilon_{y}=\int d \phi_{y}
$$

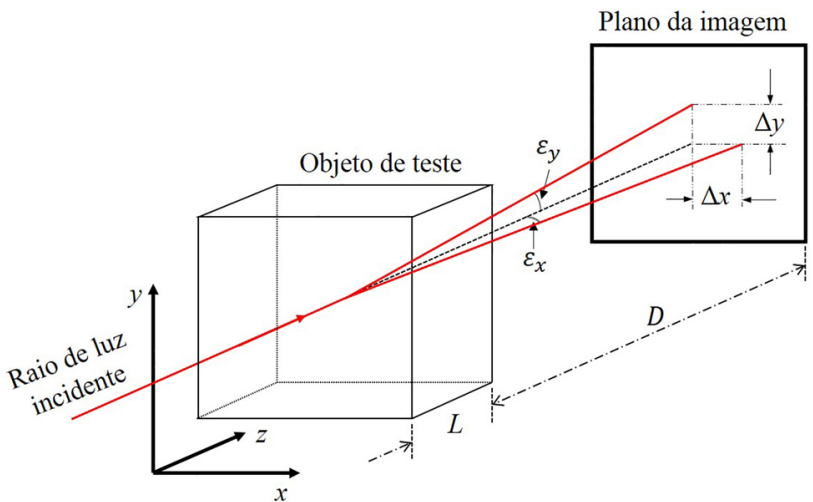

Figura 5: Deflexão de um raio de luz em um objeto de teste. Fonte: adaptada de H. W. Liepmann, A. Roshko [14].

onde a integral é tomada ao longo do raio de luz curvado, na direção $z$. A deflexão do raio é usualmente muito pequena de modo que a densidade do meio ao longo do raio curvado é a mesma que nos pontos vizinhos: $x=x_{1}$ e $y=y_{1}$. Então

$$
\begin{aligned}
& \varepsilon_{x}=\int_{0}^{L} \frac{1}{R} d z=\frac{1}{n} \int_{0}^{L}\left(\frac{\partial n}{\partial x}\right)_{x_{1}} d z \\
& \varepsilon_{y}=\int_{0}^{L} \frac{1}{R} d z=\frac{1}{n} \int_{0}^{L}\left(\frac{\partial n}{\partial y}\right)_{y_{1}} d z
\end{aligned}
$$

onde $L$ é a extensão do meio na direção $z$. Estas são relações fundamentais para meios com pequenos gradientes de densidade, como ocorre nos escoamentos isentrópicos ou escoamentos quase isentrópicos.

Se o meio apresentar as mesmas condições ao longo de todo o eixo $z$, obtém-se:

$$
\varepsilon_{x}=\frac{L}{n_{0}} \frac{\partial n}{\partial x} \quad ; \quad \varepsilon_{y}=\frac{L}{n_{0}} \frac{\partial n}{\partial y}
$$

em que $n_{0}$ é o índice de refração do meio circundante.

Se houver um plano de projeção a uma distância $D$, conforme mostrado na Figura 5, têm-se os desvios no plano de projeção iguais a $\Delta x=D \cdot \operatorname{tg}\left(\varepsilon_{x}\right)$ e $\Delta y=$ $\operatorname{Dtg}\left(\varepsilon_{y}\right)$. 
Deve-se notar que os desvios dos raios de luz causados por uma variação do índice de refração dependem exclusivamente das propriedades físicas do meio e não dependem do tipo de instrumento usado para detectar os desvios da luz. A teoria geométrica da refração é apenas uma aproximação à abordagem da óptica física mais completa, porém, é o suficiente para o propósito do presente artigo.

O princípio básico das técnicas schlieren e shadowgraph é a combinação da projeção óptica de um objeto com uma indicação da sua deflexão da luz. Nas seções a seguir serão apresentados os métodos shadowgraph e schlieren-Toepler, os mais empregados para visualização de escoamentos.

\section{O Método Shadowgraph}

Nos métodos shadowgraph atuais a imagem formada é visualizada em um anteparo ou câmera, sendo o resultado da deflexão angular sofrida pelos raios de luz na seção de teste. A posição do objeto de teste deve estar próxima ao anteparo, para maior contraste da imagem. Podem ser usadas lentes ou espelhos para aumentar a iluminação da imagem e a sensibilidade da técnica.

A Figura 6 mostra a aplicação do método shadowgraph sem o uso de lentes ou espelhos. Os raios de luz atravessam a seção de testes onde existe uma região com variação de densidade, produzindo o efeito shadowgraph. Um feixe de luz com raios de luz paralelos entra na seção de testes, onde os raios de luz sofrem deflexões maiores ou menores ao atravessar o escoamento em estudo. Essas deflexões causam convergência e divergência dos raios de luz até atingirem o anteparo. No anteparo, se forma uma região mais clara resultante da convergência de alguns raios de luz e uma região escura decorrente da divergência de outros raios de luz. Entre elas forma-se uma região normal, onde a luminosidade não é alterada pela passagem do feixe de luz na região de testes.

Na Figura 6 são mostrados gráficos da densidade $\rho$, da derivada da densidade $\frac{\partial \rho}{\partial y}$ e da segunda derivada da densidade $\frac{\partial^{2} \rho}{\partial y^{2}}$, todas variando apenas em função da variável $y$. Pode-se notar que o máximo de brilho coincide com o máximo da segunda derivada, enquanto a região mais escura corresponde ao mínimo da segunda derivada. $\mathrm{O}$ efeito shadow não depende da densidade do meio, mas sim, da convergência ou divergência dos feixes de luz. A convergência dos raios de luz é dada por $d \varepsilon / d y$ e uma vez que a deflexão $\varepsilon$ depende do gradiente do índice de refração, consequentemente a convergência dos raios de luz depende da segunda derivada da densidade. Pode-se observar a existência de regiões claras, com maior incidência de luz, coincidentes com regiões de maior curvatura, ou seja, com maiores valores da segunda derivada da densidade. Se houver variação da densidade nas direções $y$ e $z$, o desvio da luz torna-se proporcional a $\frac{\partial^{2} \rho}{\partial y^{2}}+\frac{\partial^{2} \rho}{\partial z^{2}}$.

O método shadowgraph direto é o arranjo óptico mais simples para visualização de escoamentos. Devido à sua simplicidade e ao seu baixo custo, sem a necessidade de uso de componentes ópticos, é o método mais indicado para emprego em sala de aula. Requer somente uma fonte de luz, um objeto de teste e um anteparo. A Figura 7 mostra um esquema de visualização shadowgraph direto.

Na Figura 7 a fonte de luz é uma lanterna, o objeto de teste é uma vela, e o anteparo é um quadro branco. Um raio de luz, ao passar por uma região acima da zona de combustão da vela, sofre uma deflexão angular proporcional à segunda derivada da densidade cuja variação é causada pelo gradiente de temperatura da chama, con-
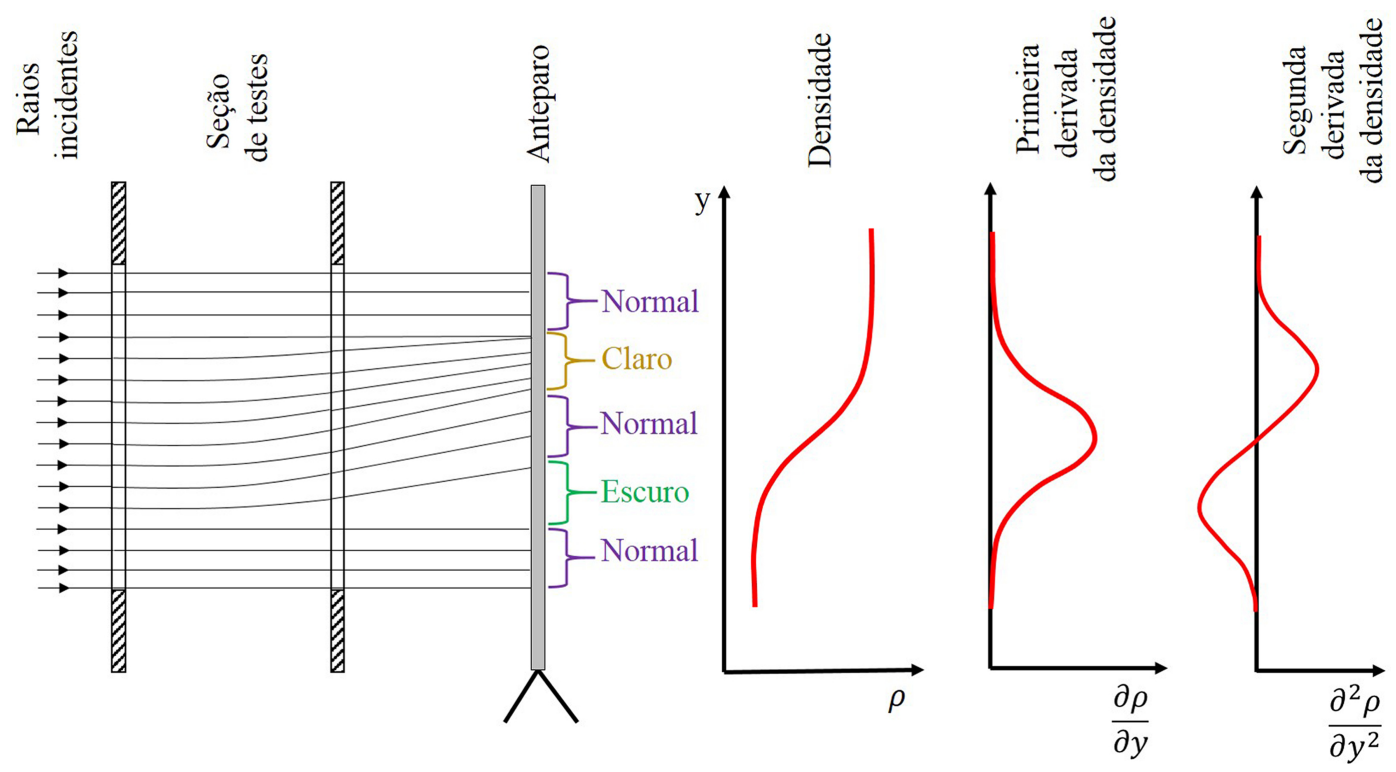

Figura 6: Efeito shadowgraph através de uma região com variação de densidade. Fonte: adaptada de H. W. Liepmann, A. Roshko [14] 


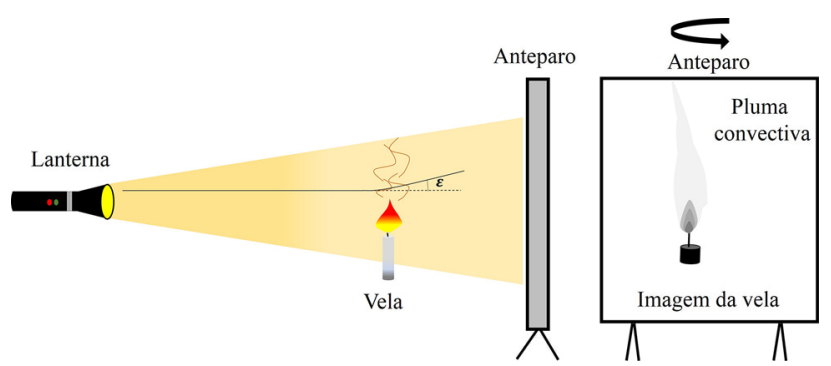

Figura 7: Esquema de um arranjo óptico shadowgraph direto. Fonte: os autores.

forme mencionado anteriormente. O raio de luz ao sofrer esse desvio não chega ao anteparo na posição que atingiria caso não houvesse o objeto de teste. A região do anteparo que deveria estar sendo iluminada, ficaria então em penumbra.

\section{O Método Schlieren-Toepler}

Os métodos schlieren fornecem um melhor contraste do campo de densidades e uma melhor iluminação do que os métodos shadowgraph. A seguir apresenta-se uma descrição do método schlieren-Toepler, o mais sensível dos métodos schlieren, que permite obter um grande detalhamento das características dos escoamentos, e possibilita visualizar escoamentos de vários tamanhos, a depender das dimensões da óptica utilizada [3], 15].

O arranjo schlieren-Toepler pode ser montado com diferentes combinações ópticas de lentes, espelhos e filtros e as características desses elementos ópticos dependem das dimensões do objeto de teste e da sensibilidade requerida.

A Figura 8 mostra um exemplo de um arranjo Toepler do tipo $Z$ (nome devido ao formato do sistema) constituído de fonte de luz, lente, fenda, dois espelhos parabólicos, faca e câmera. A faca é uma lâmina fina que serve como filtro óptico para bloquear parte dos raios refratados pelo objeto de teste.

Inicialmente, a luz emitida pela fonte é coletada por uma lente que focaliza o feixe de luz na fenda. A luz passa por esta fenda, é refletida pelo primeiro espelho e atravessa a região de teste até alcançar o segundo espelho onde é novamente refletida e focalizada junto à faca. Passa por esta e atinge a objetiva da câmera onde forma a imagem do objeto de teste, que consiste em pontos de contraste de intensidade de luz em tons de cinza.

As Figuras $9 a$ e $9 b$ mostram uma fenda que possui um ajuste de abertura para possibilitar obter uma imagem com geometria bem definida na região focal do segundo espelho, bem como propicia uma distribuição homogênea de luz nessa região. Fendas mecânicas são empregadas em arranjos em que são utilizadas lâmpadas ou LEDs como fonte de luz. As Figuras $9 c$ e $9 d$ exibem lâminas de barbear que são frequentemente usadas como faca [3].

A Figura 10 mostra a imagem da fenda localizada no foco do segundo espelho, junto à faca. O parâmetro $h$ é a altura da fenda e $b$ é a sua largura. Coloca-se a fenda na posição horizontal para destacar os gradientes de densidade vertical do objeto de teste e, vice-versa, coloca-se a fenda na vertical para destacar os gradientes de densidade horizontal do objeto de teste. A faca deve seguir a orientação da fenda, ou seja, se a fenda estiver na horizontal, o bordo da faca deve estar na horizontal, caso contrário, se a fenda estiver na vertical o bordo da faca deve ficar na vertical. Por fim, para registrar a imagem, a objetiva deve ser ajustada para se obter na câmera a imagem correspondente ao objeto inserido na área de teste.

No arranjo óptico da Figura 8, a fonte de luz é considerada um corpo extenso, portanto, a colimação não

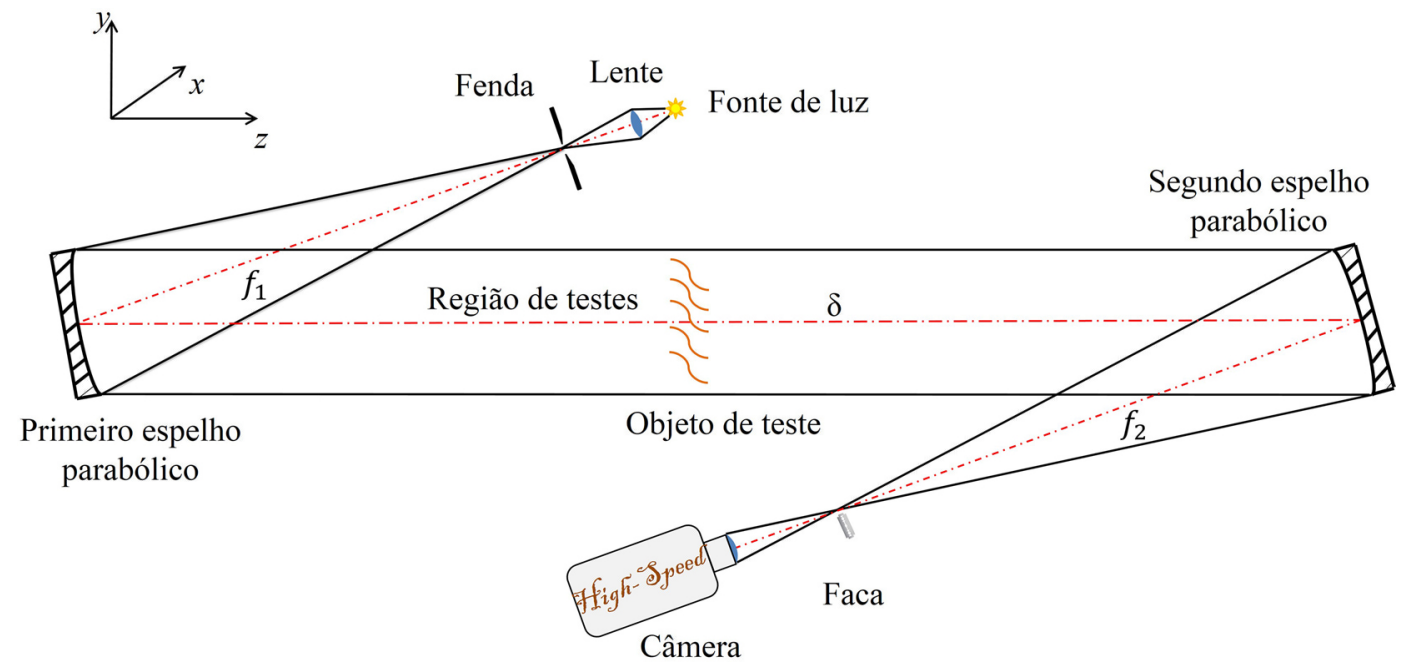

Figura 8: Arranjo óptico Schlieren-Toepler, em que $f_{1}$ e $f_{2}$ são as distâncias focais dos espelhos parabólicos e $\delta$ é a distância entre os espelhos. Na faca é formada uma imagem da fonte de luz e na câmera é formada a imagem schlieren do objeto inserido na área de teste. 
(a)

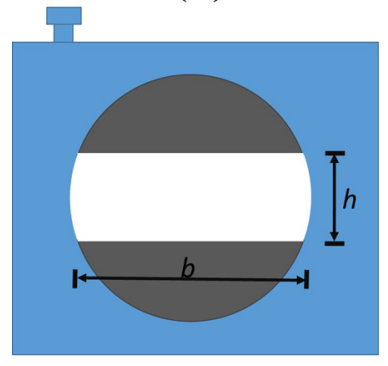

(b)

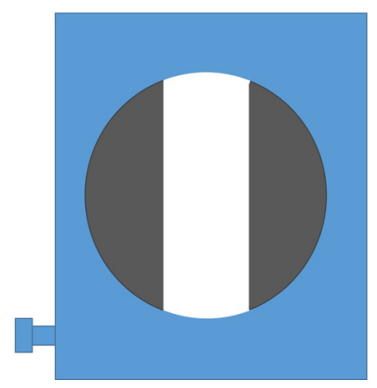

(c)

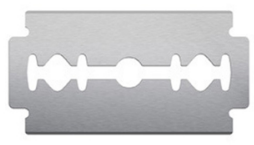

(d)

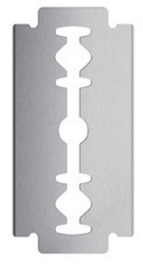

Figura 9: Componentes utilizados no arranjo Schlieren-Toepler: ( $a$ ) fenda com orientação horizontal, $(b)$ fenda com orientação vertical, $(c)$ lâmina com bordos de orientação horizontal e $(d)$ lâmina com bordos de orientação vertical. Fonte: os autores.

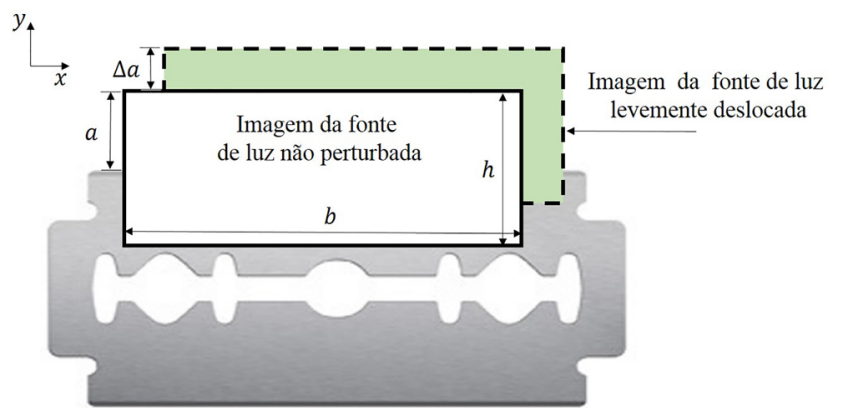

Figura 10: Diagrama do plano da faca, mostrando a imagem da fenda e da imagem deslocada da fonte de luz, ambas com dimensões $b$ eh. Fonte: os autores.

produz raios de luz exatamente paralelos, esse efeito faz com que a imagem formada da fonte de luz na faca seja uma série de pontos de luz. Como a fonte de luz tem um tamanho finito, cada ponto da área de teste é iluminado por inúmeros raios de luz, que são delimitados pelas extremidades da fonte. Portanto, cada ponto da fonte emite luz que ilumina toda a seção de teste. Além disso, as dimensões da fonte ocasionam uma profundidade de foco junto à faca no sistema schlieren. Além desse efeito, a passagem da luz pelo objeto de teste causa um deslocamento da imagem da fonte. Toepler foi o primeiro cientista a reconhecer que a imagem da fonte de luz formada na faca é a superposição de múltiplas imagens separadas de todos os pontos da seção de teste 11]. Nas Figuras $9 a$ e $9 c$ a fenda e o bordo da faca foram posicionados de forma horizontal. Nessa condição a Figura 10 mostra o diagrama do plano da faca.

$\mathrm{Na}$ Figura 10, a imagem composta da fonte (imagens com e sem deslocamento) é bloqueada pela faca. A imagem da fonte não deslocada possui altura desobstruída igual a $a$. Os raios de luz que sofreram deflexão $\varepsilon_{y}$ formam uma imagem levemente deslocada da fonte, com altura desobstruída igual a $a+\Delta a$. No caso de um gradiente de densidade reduzido na horizontal, o deslocamento horizontal do feixe de luz não contribui para o contraste da imagem. A variação da altura desobstruída da imagem da fonte é dada por:

$$
\Delta a=f_{2} \varepsilon_{y}
$$

em que $f_{2}$ é a distância focal do segundo espelho (Figura 8) e $\varepsilon_{y}$ é a deflexão perpendicular à borda da faca. A imagem formada consiste em uma matriz de elementos de imagem caracterizados por variações de contraste em uma escala cinza, decorrentes do objeto de teste, e dependente da intensidade da iluminação pela fonte.

A sensibilidade e o contraste em arranjos schlieren serão tratados a seguir conforme a abordagem de Settles 3]. A sensibilidade de um arranjo óptico Toepler tipo Z, semelhante ao da Figura 8, será determinada para o caso de uma fonte de luz e faca com orientação horizontal.

Considerando que não haja perdas de luminosidade no caminho óptico da luz entre a fenda e a câmera e com pouca inclinação dos raios luminosos, a iluminância $E_{o}$ incidente sobre o primeiro espelho pode ser calculada por meio da lei do inverso do quadrado, que é dado por:

$$
E_{0}=\frac{B b h}{f_{1}^{2}}
$$

em que $b$ e $h$ são as dimensões da fenda, $B$ é a luminância da fonte de luz que passa através da fenda (candela $/ \mathrm{m}^{2}$ ) e $f_{1}$ é a distância focal do primeiro espelho. A iluminância da imagem, considerando ausência de perdas, sem a utilização da faca e com um fator de magnificação $A$ entre a imagem e a seção de teste, é calculada por:

$$
E_{0}=\frac{B b h}{A^{2} f_{1}^{2}}
$$

A iluminância $(E)$ é a intensidade de luz incidente por unidade de área em uma direção enquanto a luminância $(B)$ é a intensidade de luz emitida por unidade de área em uma direção. No caso de obstrução parcial dos raios de luz pela faca, com uma altura desobstruída $a$, conforme a Figura 10, substitui-se $h$ na Eq. 10 por $a f_{1} / f_{2}$, e a iluminância da imagem schlieren fica:

$$
E=\frac{B b a}{A^{2} f_{1} f_{2}}
$$

A iluminância em cada ponto da imagem é avaliada por comparação à iluminância média $E$ (cinza médio): maior, 
menor, ou igual à mesma. No caso de um objeto na área de teste do arranjo schlieren, os raios de luz da fonte sofrerão um desvio angular $\varepsilon$, onde $\varepsilon_{y}$ é a componente na direção $y$ do desvio. Isto causa uma pequena variação na posição vertical da imagem da fonte $\Delta a=f_{2} \varepsilon_{y}$ em relação ao bordo horizontal da faca. Substituindo $a$ por $\Delta a$ na equação (11), obtém-se a variação de iluminância $\Delta E$ em um ponto da imagem, devida à refração $\varepsilon_{y}$ causada pelo objeto de teste:

$$
\Delta E=\frac{B b \varepsilon_{y}}{A^{2} f_{1}}
$$

O contraste $C$ na imagem schlieren é a relação de variação da iluminância $\Delta E$ com a iluminância de fundo $E$, sendo dado por:

$$
C \equiv \frac{\Delta E}{E}=\frac{f_{2} \varepsilon_{y}}{a}
$$

Substituindo as Equações (13) e (2) na equação (19), resulta:

$$
C=\frac{f_{2}}{a} \frac{L}{n_{0}} \frac{\partial n}{\partial y}=\frac{f_{2}}{a} \frac{L k}{1+k \rho_{0}} \frac{\partial \rho}{\partial y}
$$

mostrando que o contraste da imagem é proporcional ao gradiente de densidade ou massa específica do objeto de teste em um sistema schlieren.

O contraste na imagem corresponde à saída do arranjo schlieren. A entrada é um conjunto de deflexões irregulares de raios, $\varepsilon$, resultantes dos gradientes do índice de refração na área de teste, dentro de um feixe de luz que seria, de outra forma, regular. Uma vez que a sensibilidade de qualquer instrumento é basicamente um coeficiente de influência, isto é, $d($ saida $) / d($ entrada $)$, pode-se determinar a sensibilidade schlieren $(S)$ - também denominada sensibilidade de contraste - como a taxa de variação do contraste com relação ao ângulo de refração:

$$
S=\frac{d C}{d \varepsilon}=\frac{f_{2}}{a}
$$

Portanto, a sensibilidade da técnica independe de qualquer observação. Esse parâmetro é importante para planejar um arranjo óptico com a maior sensibilidade possível, dadas as características dos elementos ópticos disponíveis no laboratório. Segundo a equação (14), a sensibilidade é diretamente proporcional à distância focal do segundo espelho e inversamente proporcional à altura desobstruída da imagem da fenda.

Algumas considerações sobre os componentes ópticos do arranjo tipo Z são feitas a seguir.

É desejável o menor valor possível para a altura desobstruída da imagem da fenda. No entanto, existe um compromisso entre as dimensões da fenda e a quantidade de luz transmitida através dela. A quantidade de luz mínima deve ser suficiente para sensibilizar o sensor da câmera, a fim de que se possa visualizar a imagem do objeto de teste. Essa quantidade de luz varia de acordo com o tipo de sensor, marca e modelo da câmera, sendo que as câmeras intensified charge-coupled device (ICCD) são as mais sensíveis encontradas no mercado. Porém, para a maior parte dos experimentos, câmeras complementary metal-oxide semiconductor (CMOS), do tipo single-lens reflex (SLR) são suficientes para obtenção de imagens, por exemplo, as marcas Nikon e Cannon oferecem câmeras semiprofissionais que atendem a essas necessidades.

Uma maneira de se obter a maior quantidade de luz em um arranjo óptico Toepler é utilizar uma lente para focalização da luz proveniente da fonte com o maior diâmetro possível que não produza aberração óptica e com uma pequena distância focal. Uma típica lente utilizada possui $5 \mathrm{~cm}$ (2") de diâmetro e $10 \mathrm{~cm}$ de distância focal. As posições da fonte de luz, da lente e da fenda, podem ser facilmente calculadas pela equação de Gauss para lentes delgadas. Conforme já mencionado, a sensibilidade é proporcional à distância focal do segundo espelho. Um espelho convencional para o arranjo deve possuir no mínimo 1 metro de distância focal, pois arranjos ópticos com espelhos de distância focal abaixo de 1 metro encontram problemas de sensibilidade e dificuldade de alinhamento. A distância focal do primeiro espelho deve ser igual à do segundo espelho, também para evitar aberrações ópticas. A qualidade óptica mínima dos espelhos parabólicos deve ser $\lambda / 4$, onde $\lambda$ é o comprimento do raio da luz incidente. Espelhos parabólicos podem ser substituídos por espelhos esféricos, todavia estes produzem aberração óptica esférica. Para diminuir o efeito das aberrações, o comprimento da região de teste, variável $\delta$ na Figura 8, deve ser maior que o dobro da distância focal dos espelhos. Os ângulos entre a fonte de luz e o primeiro espelho e entre o segundo espelho e a câmera devem ser os menores possíveis, também para reduzir eventuais aberrações ópticas. Recomenda-se o uso de mesa óptica para um melhor alinhamento dos espelhos.

\section{Alinhamento Óptico do Método schlieren-Toepler}

A Figura 11 mostra uma variante do método SchlierenToepler onde não são utilizados espelhos. Este arranjo é simples de reproduzir e pode ser facilmente utilizado em sala de aula. Como fonte de luz do sistema, pode ser utilizado um LED de emissão de cor branca de 3 $\mathrm{W}$ de potência. Este modelo pode ser encontrado em lojas de eletrônica, mais conhecido como super LED com dissipador estrela. São utilizadas duas lentes convergentes, a lente próxima à fonte de luz possui $20 \mathrm{~cm}$ de distância focal e duas polegadas $(5 \mathrm{~cm})$ de diâmetro, sendo que a área de teste é limitada pelo diâmetro da lente. Posterior à região de testes encontra-se a lente focalizadora, que possui a mesma função do segundo espelho parabólico do arranjo óptico da Figura 8. A fim de produzir uma boa sensibilidade ao arranjo, a lente deve ter uma distância focal acima de 1 metro, pois a sensibilidade da técnica é proporcional à distância focal, como já observado. Após a faca, é posicionada uma tela ou câmera, onde é formada a imagem schlieren do objeto presente na área de teste. 


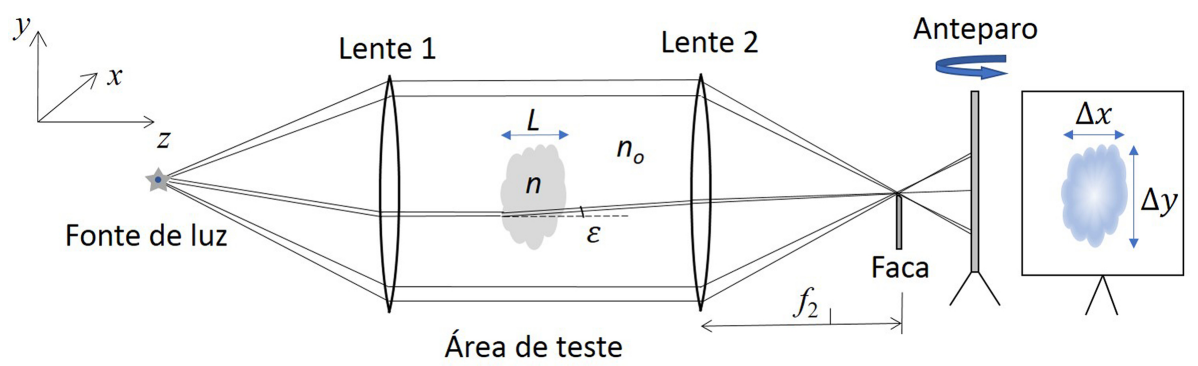

Figura 11: Arranjo Schlieren-Toepler composto por duas lentes, um filtro óptico "faca" e um super LED com dissipador estrela. Fonte: os autores.

No método Toepler o maior desafio para conseguir boas imagens é encontrar a posição correta da faca. Por outro lado, o anteparo pode ser posicionado a qualquer distância após a formação da imagem schlieren. No entanto, quanto maior a distância, maior será a ampliação da imagem e menor será a quantidade de luz por unidade de área da imagem.

A técnica Toepler possui uma grande sensibilidade na região focal e por isso deve-se ajustar a posição da faca tanto horizontal quanto verticalmente. Primeiramente, deve-se encontrar a posição horizontal, em geral com exatidão da ordem de décimos de milímetro, a fim de se obter um contraste adequado para a imagem schlieren. Uma forma prática de encontrar essa região é observando a imagem formada no anteparo. A Figura 12 mostra os efeitos do posicionamento da faca sobre imagens schlieren de um escoamento gerado por um soprador de ar quente. As Figuras $12 a$ e $12 b$ mostram imagens produzidas, respectivamente, quando a faca é posicionada antes e depois do ponto ideal. A Figura $12 c$ mostra a imagem produzida com posicionamento ideal da faca, podendo-se notar uma iluminância de fundo homogênea e os detalhes de todo o escoamento inserido na região de teste.

Após encontrar a posição horizontal correta para a faca, o próximo passo é ajustar verticalmente a posição da faca, o que definirá a porcentagem do feixe de luz a ser obstruída. Pode-se relacionar a sensibilidade da técnica (a)
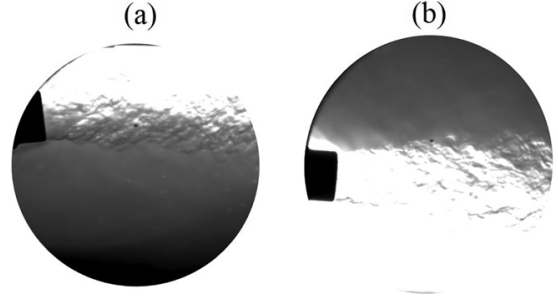

(c)

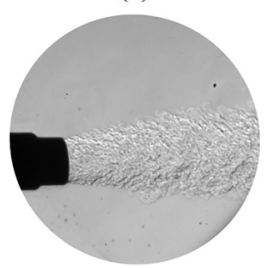

Figura 12: Imagens schlieren de um escoamento produzido por um soprador de ar quente: $(a)$ imagem com faca colocada antes da região focal; $(b)$ imagem com faca colocada depois da região focal; $(c)$ imagem com faca colocada na posição correta, imagens obtidas utilizando o arranjo Schlieren-Toepler da Figura 8. Fonte: os autores. com a porcentagem de luz cortada por:

$$
S=\frac{f_{2}}{a[1-\gamma / 100]}
$$

em que $\gamma$ é a porcentagem do feixe de luz obstruído pela faca.

Na Figura 13 são mostradas imagens schlieren de um escoamento supersônico, produzido a partir da saída de uma mangueira acoplada em um compressor de ar, em que foi variada a porcentagem de luz obstruída pela faca. Na Figura 13(a) foram obstruídos aproximadamente 30\% da iluminância na região focal, enquanto que nas Figuras $13(b)$ e (c) foram obstruídos $50 \%$ e $70 \%$, respectivamente. Pode-se observar que o contraste é maior para o caso de $70 \%$. Contudo, de acordo com a equação (22), para uma obstrução de $90 \%$, é esperada uma sensibilidade maior na imagem. Porém existe um compromisso entre a sensibilidade de detecção da câmera, ou os olhos do observador, e a quantidade de luz bloqueada. Uma câmera comum possui uma sensibilidade à luminosidade semelhante ao olho humano. Portanto, para fins práticos, em geral, acima de $90 \%$ de obstrução, a resposta deixa de ser adequada tanto para uma câmera comum quanto para o olho humano.

A faca funciona como um filtro espacial passa alta, bloqueando parte da iluminância $E$ dada pela equação (17), e permitindo a passagem dos raios de luz refratados, parâmetro $\Delta E$ da equação $(18)$. Tendo em vista que o contraste dado pela equação $(19)$ é proporcional a $\Delta E$ e inversamente proporcional a $E$, é desejável que a faca atenue o máximo possível os raios de fundo, porém, como pode ser observado pela Figura 13 e discutido acima, existe o fator limitante que é a sensibilidade de detecção.

\section{Resultados e Discussões do Método Schlieren}

$\mathrm{Na}$ visualização de escoamentos não reativos das Figuras 14, 15 e 16 foi utilizado um arranjo Schlieren-Toepler semelhante ao da Figura 8. Porém, como alternativa ao emprego de espelhos parabólicos que são demasiadamente caros, foram utilizados espelhos esféricos. Estes foram fornecidos pela empresa Edmund Optics que oferece kits para sistemas schlieren com preços inferiores a US\$1000,00 17. O kit contém 2 espelhos esféricos de 
6 polegadas com distância focal de $1,52 \mathrm{~m}$ e qualidade óptica de $(\lambda / 8)$, além de uma fonte de luz e um filtro óptico. O filtro óptico e a fonte de luz do kit foram substituídos por uma lâmina de barbear e um super LED de 3 W, com dissipador estrela, pois esses produzem melhores resultados. Para aquisição das imagens foi utilizada uma câmera D7100 da Nikon equipada com uma objetiva de lente intercambiável Nikkor AF 50MM. A câmera é utilizada para registro das imagens, entretanto um anteparo pode substituí-la para visualização das imagens schlieren. a) $30 \%$

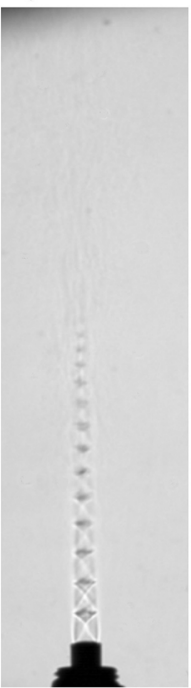

b) $50 \%$

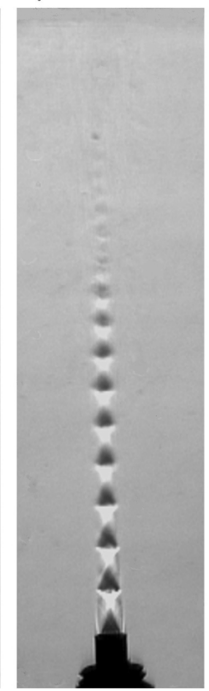

c) $70 \%$

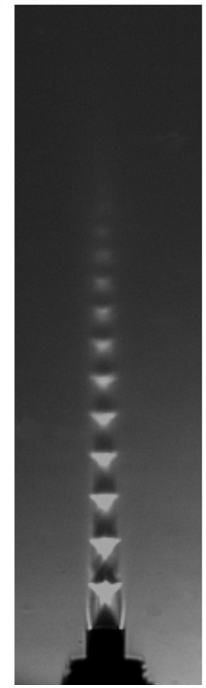

d) $90 \%$

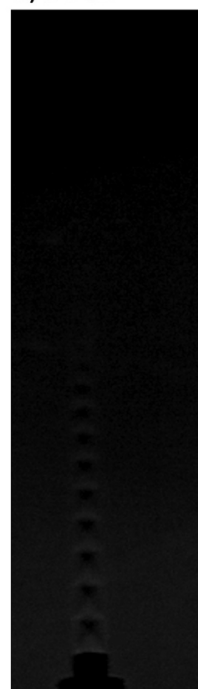

Figura 13: Escoamento supersônico de ar atmosférico, onde foi variada a porcentagem de luz cortada, imagens obtidas utilizando o arranjo Schlieren-Toepler da Figura 8. Fonte: os autores.

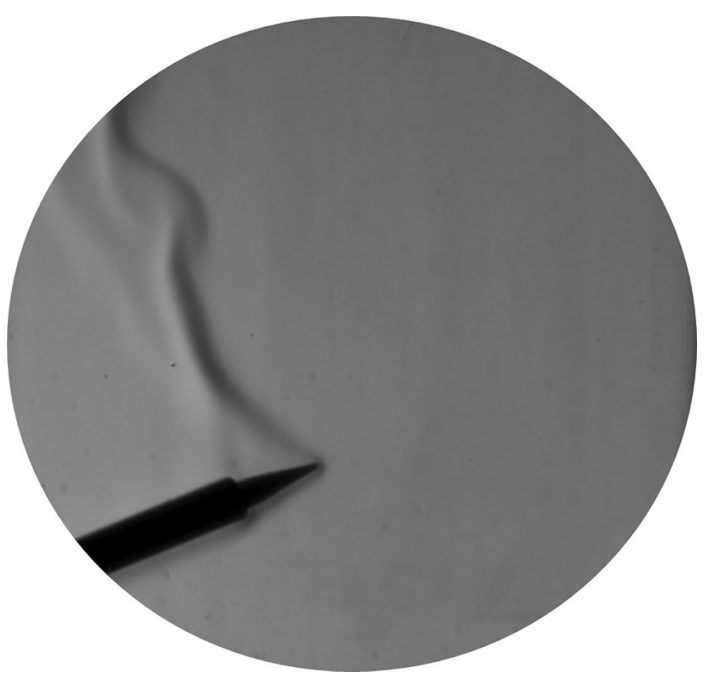

Figura 14: Imagem do escoamento convectivo de ar aquecido produzido por um ferro de solda, obtida com um arranjo SchlierenToepler do tipo $Z$. Fonte: os autores

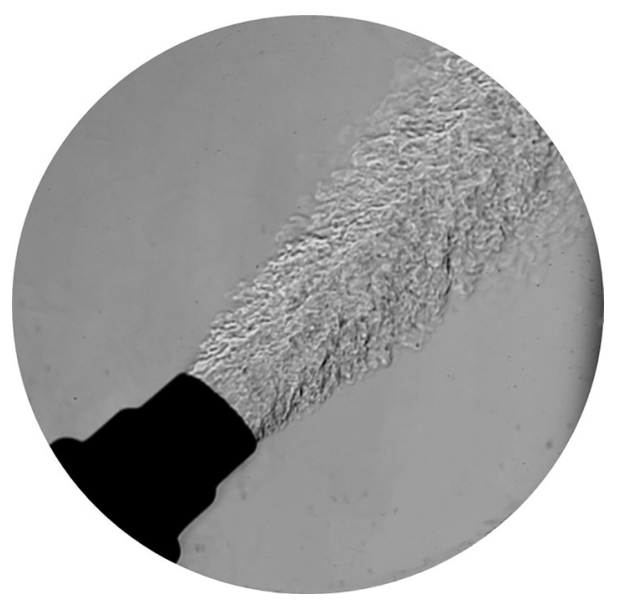

Figura 15: Imagem do escoamento turbulento de ar aquecido produzido por um secador de cabelo, obtida com um arranjo Schlieren-Toepler do tipo $Z$. Fonte: os autores.

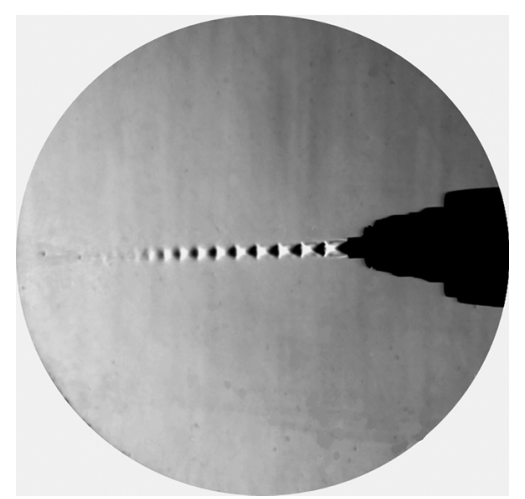

Figura 16: Imagem do escoamento supersônico com ondas choque e expansão, produzido por uma mangueira de ar comprimido, obtida com um arranjo Schlieren-Toepler do tipo Z. Fonte: os autores.

\subsection{Imagens Schlieren}

Na Figura 14 é mostrada a imagem de um ferro de solda aquecido e o escoamento resultante produzido pelo gradiente de temperatura. A transferência de calor entre o ferro de solda e o meio circundante causa aquecimento e convecção do ar, o que leva a uma alteração no seu índice de refração, possibilitando assim a sua visualização schlieren. A convecção é o deslocamento do ar devido à força de empuxo gerada pela redução da densidade do ar causada por seu aquecimento.

Na Figura 15 é mostrada a imagem de um escoamento turbulento aquecido produzido por um secador de cabelo. Este possui um filamento resistivo quente, junto ao qual passa o fluxo de ar que sofre aumento de sua energia térmica (entalpia). A combinação dos gradientes de pressão e temperatura no escoamento produz uma estrutura de grande turbulência, podendo-se observar, na Figura 15, os vórtices formados.

A Figura 16 mostra a imagem de um escoamento supersônico produzido por uma mangueira de ar, empregandose um compressor de ar comercial. Pode-se observar a 


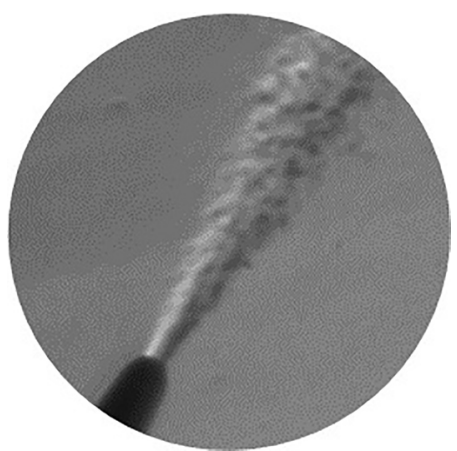

Figura 17: Escoamento de acetona difundido em meio ambiente, imagem obtida utilizando o arranjo Schlieren-Toepler linear composto apenas por lentes. Fonte: os autores.

formação de uma estrutura com perfil de diamantes, constituída por ondas de choque e ondas de expansão.

A Figura 17 mostra a imagem de um escoamento difusivo de vapor de acetona liberada por uma pisseta. A equação 200 mostra que o contraste na imagem é proporcional ao gradiente do índice de refração do escoamento de acetona e inversamente proporcional ao índice de refração do ar. O vapor de acetona possui $n=1,001090$ e o ar $n=1,000293$. Esta variação está na terceira casa decimal, sendo uma das maiores encontradas na natureza para gases. O valor do índice de refração de um gás é proporcional à sua massa molar, de acordo com a equação (3). Considerando que o vapor de acetona está em uma mesma temperatura e pressão que o ar circundante, pode-se dizer, em última instância, que a visualização do vapor de acetona é possível graças à sua massa molar.

Para aquisição das imagens da Figura 17, foi utilizado o arranjo óptico descrito na Figura 9, composto apenas por lentes. Para aquisição das imagens foi utilizada a mesma câmera do arranjo tipo $Z$. As imagens foram obtidas com a câmera operando no modo monocromático e devido às distâncias focais reduzidas das lentes usadas, a imagem não obteve um contraste adequado.

\section{Conclusões}

Este trabalho descreveu os fundamentos e aplicações dos métodos ópticos shadowgraph e schlieren utilizados em estudos de aerodinâmica e de escoamentos, visando o desenvolvimento de carros, aviões, foguetes e outros equipamentos. Esses métodos ópticos apresentam simplicidade construtiva, baixo custo e grande sensibilidade em relação a outros métodos de diagnóstico. A utilização de câmeras de alta velocidade e de alta resolução, juntamente com o emprego de softwares de processamento de imagens, permitiu um desenvolvimento acentuado dos métodos schlieren e shadowgraph. Esses métodos se tornaram quantitativos, permitindo determinar a geometria e as distribuições de densidades, velocidades e temperaturas de escoamentos inertes ou reativos, internos ou externos. O método shadowgraph direto foi apresentado como uma aplicação simples e de baixo custo para em- prego em sala de aula. Foi também descrito em detalhes o método schlieren-Toepler, base de métodos schlieren quantitativos. Esse método schlieren é o mais utilizado por apresentar maior contraste e sensibilidade dentre os métodos schlieren, além de facilidade de alinhamento e possibilidade de observar regiões de teste de diferentes tamanhos.

\section{Agradecimentos}

Os autores agradecem à FINEP e à FAPESP pelo apoio financeiro ao projeto e à CAPES pela concessão de uma bolsa de doutorado ao primeiro autor.

\section{Referências}

[1] M.S.T. Araújo e M.L.V.S. Abib, Revista Brasileira de Ensino de Física 25, 176 (2003).

[2] M. Muller, J.L. Fabris, R. Falate e E. Cação Jr., Revista Brasileira de Ensino de Física 25, 273 (2003).

[3] G.S. Settles, Schlieren and shadowgraph techniques: visualizing phenomena in transparent media (Springer-Verlag, Berlin, 2000).

[4] A.C. Avelar, E. Basso, J.B.P. Falcão Filho, C.P.F. Francisco e P.G.M. Romero, J Braz. Soc. Mech. Sci. Eng. 40, 130. (2018).

[5] G.S. Settles e M.J. Hargather, Meas. Sci. Technol. 28, 042001 (2017)

[6] G.E. Elsinga, Density measurements by means of schlieren methods. Dissertação de Mestrado, Delft University of Technology, Delft (2003).

[7] C.T. Wanstall, A.K. Agrawal e J.A. Bittle, Applied Optics, 56, 8385 (2017)

[8] M.J. Hargather, M.J. Lawson, G.S. Settles e L.M. Weinstein, AIAA Journal 49, 611 (2011).

[9] S. Biswas, Physics of Turbulent Jet Ignition (Springer Thesis, West Lafayette, 2018).

[10] A.W. Gena, C. Voelker e G.S. Settles, Indoor Air, $32302432(2020)$.

[11] P. Krehl e S. Engemann, Shock Waves 5, 1 (1995)

[12] A. Mazumdar, Principles and techniques of schlieren imaging systems (Columbia University, New York, 2013)

[13] H.W. Liepmann e A. Roshko, Elements of Gasdynamics (Wiley, New York, 1957).

[14] C.L. Yaws, Gas Data book (McGraw-Hill, New York, 2001).

[15] L.A. Vasil'Ev, Schlieren Methods (Keter Publishers House, Jerusalém, 1971).

[16] L. Fassarella, Revista Brasileira de Ensino de Física 29, 215 (2007).

[17] https://www.edmundoptics.com/f/schlierensystems/11889/, acessado em 03/06/2020. 